\title{
CONCEPTO Y RESPONSABILIDAD DEL NAVIERO ANTE LA REFORMA DEL CODIGO DE COMERCIO ${ }^{1}$
}

\author{
Dr. Rafael Matilla Alegre \\ Catedrático de Derecho Mercantil de la Universidad de Deusto
}

\begin{abstract}
Sumario: Concepto de naviero. El concepto en el Código de Comercio. La noción en otras normas. El concepto y las normas sobre inscripción del naviero. Relación entre los conceptos de buque y de naviero. Derecho de la navegación y la noción de naviero. Responsabilidad del naviero: 1. En el Código de Comercio; 2. En el Código Penal; 3. En la Ley de Puertos del Estado y la Marina Mercante.
\end{abstract}

\section{Concepto de naviero}

La doctrina entiende que el naviero es el empresario marítimo, la persona que explota empresarialmente un buque (o en cuyo nombre se explota) y, también, que el régimen jurídico establecido en el Código de Comercio es muy confuso (se ha afirmado incluso que no ha podido ser precisado), y que es necesario diferenciar los conceptos de naviero y propietario del buque.

El naviero puede ser en efecto el propietario del buque y lo es en muchos casos; pero puede disponer del buque por otro título. Esta situación última no era frecuente en otras épocas, pero sí actualmente al haberse hecho usuales en el tráfico marítimo los contratos de arrendamiento de buque en sus dos modalidades de arrendamiento a casco desnudo y de un buque armado y equipado y de fletamento en time charter.

La distinción entre propietario y naviero estaba recogida en el Derecho Romano donde se diferenciaban los conceptos de propietario del bu-

\footnotetext{
1 Ponencia presentada en el Congreso Internacional de Derecho Marítimo celebrado en Bilbao para conmemorar el décimo aniversario de la fundación de la Escuela de Administración Marítima por el Gobierno del País Vasco.
} 
que, de exercitor navis y de nauta o señor de la nave. El exercitor navis era el dominus negoti, que explotaba el buque en nombre propio y por su cuenta haciendo de su cargo las pérdidas y beneficios correspondientes; podía ser el propietario del buque, pero no lo era en todo caso.

El magister navis (nauta o señor de la nave) era quien la explotaba directamente nombrado por el exercitor navis; y éste respondía por su actuación y por la explotación del buque en función de la acción exercitoria.

En la Edad Media, el Libro del Consulado del Mar recoge y reglamenta la figura del senyor de la nau, que se embarca en la nave, dirige la expedición (a veces, auxiliado por un técnico en navegación, nauxer), tiene a su cargo, orden y mando, el buque y la tripulación, bajo un régimen específico de responsabilidad propia.

El senyor de la nau podía ser el propietario o un tercero, pero en este caso, aunque actuase en nombre propio, lo hacía por cuenta del propietario o copropietarios del buque; o al haberlo recibido en depósito en función de un contrato de commenda, por cuenta propia y del comendator propietario del buque... o por cuenta de un conjunto de colonnistas asociados para la expedición marítima y por tanto en interés del conjunto de los partícipes en una de las fórmulas asociativas usuales en la época bien distintas de los contratos de explotación del buque frecuentes en el tráfico marítimo actual.

Las Ordenanzas marítimas francesas de 1681, en una época en que ya están diferenciadas las actividades comerciales terrestres y marítimas, distinguen las nociones de propietario (y copropietarios del buque) y de capitán, éste un representante de aquél o de éstos. En estas Ordenanzas, el capitán respondía personalmente de la custodia y entrega del cargamento y en general de las obligaciones que pudiesen resultar de la navegación y explotación del buque, y el propietario o copropietarios, por la conducta del capitán, aunque limitadamente si hacían abandono del buque y flete. Pero las Ordenanzas de 1681 no diferenciaban los conceptos de propietario y naviero, dando por supuesto que el buque era explotado por el propietario.

Y del mismo modo en las Ordenanzas de Bilbao (cap. 24).

La distinción no está recogida tampoco en el Código de Comercio Francés de 1807, que no se refiere sino a los propietarios del buque (y al capitán), a decir de los comentaristas, porque el Código no ha previsto que el buque pueda ser explotado por quien no sea su propietario.

La distinción aparece por primera vez con el Código alemán de 1897, al admitir que puede ser naviero quien emplea un buque ajeno en el tráfico comercial marítimo por cuenta propia. 


\section{El concepto en el Código de Comercio}

El Código de Comercio distingue los conceptos de propietario y de naviero (arts. 586, 588 y 595 en que habla de ambos como personas diferentes) y califica al naviero de comerciante marítimo, exigiéndole que tenga aptitud para comerciar y que se halle inscrito en la matrícula de comerciantes de la provincia (hoy, en el Registro Mercantil); inscripción obligatoria como es sabido (art. 595).

Y configura al naviero, en opinión bastante usual, como comerciante marítimo, que explota un buque de transporte o pasajeros; como centro de esta actividad comercial (hoy, más bien, empresarial), desde el momento en que gestiona judicial y extrajudicialmente cuanto interesa al comercio; al hacerle responsable de las obligaciones contraídas por el capitán y de los actos de éste (arts. 586, 587, 588) y de las que puedan resultar en los casos de abordaje (arts. 826 y 837)..., y al facultarle para fletar el buque y nombrar la tripulación...

Pero, con esta noción y con este concepto, no es posible explicar satisfactoriamente el sistema legal en determinados extremos básicos:

En primer término, porque el Código define al naviero como la persona encargada de representar y avituallar el buque en el puerto en que se encuentre (art. 586) y, también, y en esta línea, porque recoge la figura del naviero gestor (al que se exige asimismo aptitud para comerciar e inscripción en el Registro (art. 595), que no es sino un apoderado general (arts. 597 y 598) nombrado necesariamente en los casos de copropiedad naval... Incluso parece identificar a veces las nociones de propietario y naviero.

En segundo lugar, porque al propietario (que, en este criterio interpretativo, puede no ser naviero) se le hace responsable y deudor de las obligaciones contractuales del capitán que no se haya excedido en las funciones que le corresponden (arts. 586 y 588 entre otros) y de los actos del capitán (art. 586)...; es decir, se le hace responsable de la actuación del capitán (y de la tripulación), siendo así que es un auxiliar del naviero nombrado por él, que ha de seguir sus intrucciones... (arts. 597, 610...); y se da por supuesto que ha de soportar la afección real del buque en favor de los acreedores marítimos privilegiados del art. 580 .

Ni cabe explicar tampoco que el Código faculte a un naviero no propietario, ni gestor del propietario, a limitar su responsabilidad por las indemnizaciones a que diere lugar la conducta del capitán en la custodia de los efectos que cargó en el buque mediante el abandono de éste (art. 587); esto es, a concretar la responsabilidad ante tales acreedores en un bien que no le pertenece y del que no puede disponer. 
Pero hay una segunda línea interpretativa del Código (que parece más fundada):

El Código de Comercio de 1829, como los de la época, no tuvo en cuenta las formas actuales de explotación del buque que no eran usuales, ni la distinción entre propietario y naviero, si entendemos por naviero a quien explota el buque en su nombre y bajo su responsabilidad en interés y por cuenta propios.

Y esta es la situación del Código de Comercio actual, como se deduce de la Exposición de Motivos en la que dice: «Sabido es que el dominio del buque puede pertenecer a cualquiera ya sea español o extranjero, mayor o menor de edad, aunque no sea comerciante. Todas estas personas tienen aptitud para poseer y adquirir buques. Pero no la tienen para dirigir la explotación de los mismos, o sea para emplearlos o utilizarlos en especulaciones mercantiles. Por eso, desde los tiempos más antiguos, la costumbre de los pueblos primitivos ha sido poner al frente de todo buque que trate de emprender la navegación una persona caracterizada y adornada de ciertos requisitos, la cual se constituye en responsable de todos los actos y operaciones relacionados directa o indirectamente con el buque. Esta persona puede ser el propietario, si quiere y reúne condiciones legales, o un tercero a quien el primero confiere el mandato o comisión de administrar la nave en su nombre por todo el tiempo que dure el viaje».

Para la normativa del Código de Comercio, que se corresponde con la situación de la navegación en la época, el naviero es el propietario del buque que lo explota directamente o un naviero gestor de un propietario o de una comunidad de propietarios; $y$, como tal, la persona encargada de representar o avituallar el buque en el puerto en que se encuentre; el comerciante marítimo que en nombre propio gestiona judicial y extrajudicialmente cuanto interesa a la navegación.

El Código exije al naviero, y no al propietario, aptitud para comerciar y que se halle inscrito en el Registro de comerciantes de la Provincia (art. 595), siguiendo lo que era en él norma general. Porque, a tenor del Código, los comerciantes han de tener capacidad para ejercer el comercio y no simplemente capacidad jurídica (arts. 1, 4 y 5). Y, de ahí, y por eso, que el Reglamento del Registro Mercantil de 1911, y anteriormente el provisional de 1885, dispusiesen que debían inscribirse como comerciantes individuales los tutores de menores e incapacitados que en representación de éstos continuasen el comercio que hubiesen ejercido sus padres... y no dichos menores o incapacitados (arts. 92 y 95), a diferencia de lo que han dispuesto posteriormente el Reglamento de 1956 y el actual de 1989.

Y así se explica que el Código hable expresamente de naviero gestor; que parezca identificar a veces los conceptos de naviero y propietario; el 
concepto de naviero del art. 586 y que el 595 diga que representará la propiedad del buque y que pondrá en nombre propio y con tal carácter gestionar judicial o extrajudicialmente cuanto interese a la navegación,... y todos los extremos a que anteriormente se hizo referencia.

En todo caso, lo que sí parece incuestionable es que hay que modificar la normativa del Código y adaptarla a la situación actual del tráfico marítimo.

\section{La noción en otras normas}

A diferencia del Código de Comercio, la noción actual de naviero está recogida, aunque no siempre correctamente, en otras normas posteriores.

No lo está en la Ley de Hipoteca Naval de 20 de agosto de 1893 que se atiene al concepto del Código y entiende que naviero es el propietario del buque (art. 16) o el naviero gestor del propietario o copropietarios (art. 5).

Por el contrario, la Ley de 22 de diciembre de 1949, sobre transporte internacional de mercancías en régimen de conocimiento de embarque, está al concepto del Código al señalar que naviero es el propietario del buque que lo pertrecha, dota y avitualla, explotándolo por su cuenta y riesgo, y, también, el naviero gestor, esto es, la persona encargada de representar el buque donde éste se halle, pero diferenciando los conceptos de naviero y armador para entender que el armador es quien explota en nombre propio y por cuenta propia un buque ajeno del que dispone como arrendatario, esto es, el naviero no propietario del buque.

El concepto, sin embargo, está recogido en diversas normas administrativas: en primer término, en las que han regulado sucesivamente el abanderamiento, matrícula del buque y Registro marítimo (Decretos de 20 de junio de 1968; de 9 de diciembre de 1977, éste sobre abanderamiento provisional, y de 28 de julio de 1989); y, también, aunque no exclusivamente, en la Ley de Puertos del Estado y Marina Mercante de 24 de noviembre de 1992.

Las primeras, al disponer que han de inscribirse en el Registro de Empresas Marítimas las personas naturales o jurídicas propietarias de uno o más buques... y las que no siendo propietarias se dediquen a su explotación (art. 10 del D. de 1989), y al regular el abanderamiento provisional de buques españoles en el extranjero y a la inversa y exigir la presentación de los contratos de arrendamiento en los que conste que la gestión comercial y la gestión náutica del buque quedan en manos de personal no español o español respectivamente (arts. 12 y 60 D. de 1989 y D. de 1977, punto 1). 
Y la Ley de Puertos del Estado y Marina Mercante al señalar que naviero o empresa naviera es la persona física o jurídica que, utilizando buques mercantes propios o ajenos bajo cualquier modalidad admitida por los usos internacionales, se dedica a su explotación aun cuando ésta no constituya su actividad principal.

Una noción, la de las normas administrativas, que identifica los conceptos de empresario y empresa, y una noción que incluye una precisión, que se es naviero con independencia de que la actividad de explotación del buque sea o no la actividad empresarial principal.

Es decir, que hay una razón más para reclamar la modificación del concepto del Código, la necesidad de estar a un concepto único para todo el Ordenamiento Jurídico.

\section{El concepto y las normas sobre inscripción de naviero}

En el propio Código de Comercio, en las normas que contiene relativas al Registro (redactadas por la Ley de 25 de julio de 1989 de Reforma de la Legislación Mercantil), y en las del Regalmento (ahora, de 29 de diciembre de 1989), el naviero es un empresario; mejor, un empresario marítimo (hay que entender, un empresario que explota uno o varios buques): persona individual o sociedad mercantil; y, en el primer caso, aún menor de edad o incapacitado. (arts. 16, 19 y 22 del C. de c y 2, 81, 87, 88 y 91 del R.).

Estas normas no parecen requerir, ni presuponer, que el naviero sea el propietario del buque, ni que ha de tener capacidad para ejercer el comercio, ni se refieren en ningún caso al naviero gestor; es decir, que parecen estar al concepto actual del naviero.

A la vista de esta normativa (y sin entrar en el tema de la limitación de responsabilidad del naviero y con relación a él de la pérdida del derecho a limitar la responsabilidad en los casos de no inscripción, que son objeto de otra ponencia), pudiera parecer que no hace falta introducir modificación substancial alguna en el tratamiento registral del naviero y, sin embargo, no cabe compartir esta conclusión al menos por las razones siguientes:

En primer término, porque, en el Registro Mercantil, se inscribe la propiedad del buque, los gravámenes de cualquier tipo que pesan sobre él, los derechos reales constituidos sobre la propiedad del buque y limitaciones que afecten a ésta (art. $22 \mathrm{del} \mathrm{C}$. de C. en su redacción anterior a 1989 y arts. 145 y 147 del R. de 1956); pero no, los de arrendamiento de buque en ninguna de las modalidades conocidas, que son precisamente los títulos usuales del naviero no propietario. (No entramos en la dispari- 
dad terminológica existente entre los citados preceptos y otros del Código y de la Ley de Hipoteca Naval en que se utilizan términos más amplios; y no lo hacemos, porque no parecen permitir la inscripción registral del arrendamiento de buque en ningún caso).

Es decir, que, con relación a un buque concreto, podemos saber registralmente quién es su propietario y los gravámenes y derechos limitativos que pesan sobre el mismo, pero no (salvo supuestos de usufructo) si es explotado por un tercero, ni quién es el naviero cuando no lo sea el propietario. Y hay una consecuencia adicional que no cabrá entender aplicable la presunción de que se parte expresamente en determinadas legislaciones, de que el naviero es el propietario salvo inscripción registral de aquél.

Y, sin embargo, en el Registro de empresas marítimas regulado entre otras disposiciones por la Ley de Puertos del Estado y de la Marina Mercante, de 24 de noviembre de 1992, sí constan tales extremos, porque en él, con relación a cada empresario marítimo, han de hacerse constar los buques de su propiedad y los que explote que no lo sean.

En segundo lugar, porque la comunidad naval, con independencia de que no tenga reconocida expresamente personalidad jurídica, es una forma asociativa adecuada para desarrollar una actividad empresarial marítima explotando el buque que constituya su objeto, pero una forma asociativa que no tiene acceso al Registro en cuanto tal.

Y, también, porque hay que modificar el régimen jurídico aplicable, de conformidad con los caracteres históricos de la institución, tal como han sido recogidos en Derecho Comparado, para diferenciar estos supuestos de comunidad naval, que presuponen realmente un origen contractual, de las simples situaciones de copropiedad, y para exigir la división de la copropiedad en un conjunto de partes, cada una por un mismo valor; un requisito éste indispensable, incluso actualmente, en la aplicación de los preceptos legales del Código de Comercio relativos a la institución.

Y, por último, porque, modificado el art. 16 del Código de Comercio, no aparecen directamente incluidas, entre las personas jurídicas sujetas a inscripción, algunas que claramente pueden ser navieros (una circunstancia del mayor interés, porque la inscripción del naviero es obligatoria y porque la no inscripción supone un conjunto de consecuencias jurídicas); éste es el caso, de las cooperativas que exploten empresarialmente un buque y del propio Estado o de un Organismo autónomo. Un tema que no se presentaba con la redacción anterior del art. 16, porque, con arreglo a ella, el Registro tenía por objeto la inscripción de cualesquiera personas o entidades naturales o jurídicas, públicas o privadas, que se dedicasen habitualmente al comercio o cuando realizasen actos o poseyeran bienes sujetos a inscripción según las leyes o Reglamentos. 
Conviene recordar por último que, con la Ley de Puertos del Estado y de la Marina Mercante, las empresas navieras lo son aun cuando la explotación del buque no constituya su actividad principal.

\section{Relación entre los conceptos de buque y naviero}

Como se ha dicho, se es naviero por el hecho de explotar empresarialmente un buque, de donde resulta que el concepto es función de la noción de buque.

El concepto de buque no está en el Código de Comercio, y no lo estaba tampoco en el Código de Napoleón, a diferencia de otros códigos más actuales como el de la Navegación; en éste, son buques las construcciones destinadas al transporte, esto es las construcciones que pueden navegar (con medios de autopropulsión o sin ellos), pero con independencia de la finalidad del transporte, empresarial o no, aun cuando no sean propiamente buques destinados específicamente al transporte de mercancías o de pasajeros sino buques de remolque, de pesca, deportivos...

Propiamente, como el Código de Comercio español no regula sino la actividad empresarial de transporte de mercancías o de pasajeros, parece estar a esta noción restringida, que es, por otra parte, aquélla a que están la normas comunitarias y singularmente la Propuesta de Reglamento de la Comisión, de 1989, fijando los criterios para establecer una definición común de armador comunitario, en la que se entiende por tal la persona física o jurídica que preste un servicio de línea regular o esporádico en el sector del transporte marítimo de pasajeros o mercaderías por medio de uno o varios buques de su propiedad o fletados por un fletamento a casco desnudo, por viaje o por tiempo.

En nuestro Ordenamiento Jurídico la noción está en el Reglamento del Registro Mercantil de 1956 (art. 146) (y anteriormente en el de 1919) y lo está con carácter general a los efectos del Código y del Reglamento. (Un precepto y, en general todos los relativos al buque, que continúan transitoriamente en vigor tras la publicación del Reglamento de 29 de diciembre de 1989).

El Reglamento de 1919 decía que se reputaban buques no sólo las embarcaciones destinadas a la navegación de cabotaje o altura, sino también los diques flotantes, pontones, dragas, gánguiles y cualquier otro aparato flotante destinado a los servicios de la industria o comercio marítimo; y, el actual de 1956 del mismo modo, aunque cambiando el último inciso, que ahora dice destinados o que puedan estar destinados a los servicios de la industria o del comercio marítimo o fluvial. 
El concepto incluye, pues, todos lo buques con cubierta o sin ella, cualquiera que sea su tonelaje y eslora y demás características técnicas, sin estar a las distinciones usuales en la práctica y normas administrativas; estén destinados a la navegación portuaria o extraportuaria...; y no sólo los que estén afectos a una actividad empresarial de transporte en sentido estricto, sino también los que estén a una actividad empresarial distinta como puede ser la pesca, remolque...

Más aún, a tenor de esta noción, los buques no lo son sólamente cuando están destinados a una actividad empresarial, sino también cuando puedan estarlo; inciso que ha de entenderse restrictivamente (y que habría que suprimir probablemente), para considerar comprendidos en el concepto únicamente aquéllos que no estén afectos al ejercicio de una actividad empresarial, pero que lo estén por sus características; y no sin más otros como los de pesca deportiva o de recreo. (Una interpretación, por otra parte, que encuentra apoyo en las normas administrativas).

Pero el concepto es excesivamente amplio en dos aspectos, que deberán ser corregidos en una reforma posterior de nuestra legislación marítima:

En primer lugar, porque comprende no sólo las construcciones que pueden navegar, sino también los aparatos flotantes (llamados por las normas administrativas artefactos navales y plataformas fijas (art. 8 de la Ley de Puertos del Estado y Marina Mercante), aunque estén destinados a permanecer en un punto fijo, como puede ser el caso de los pontones, diques flotantes, plataformas de exploración o explotación petrolíferas... Una extensión a que no están las normas administrativas, ni el Derecho Comparado interno e internacional (por ejemplo, el Reglamento para prevenir abordajes en el mar), y, también, porque los buques son bienes muebles y estos artefactos navales, inmuebles (art. 334 del Cc.).

Y, en segundo lugar, porque, en su redacción actual, el Reglamento comprende los buques destinados al servicio de las actividades empresariales fluviales. Una referencia que no puede comprender sino los buques que desarrollen una actividad mixta, o, a lo más, los que sean susceptibles de navegar por mar; pero no, las embarcaciones fluviales propiamente dichas. Por dos razones básicas: primero, porque la navegación fluvial y la navegación por mar suponen normalmente (y parece que debe continuar siendo así) dos sistemas normativos diferentes y, segundo, porque los buques no pueden ser inscritos en el Registro Mercantil sin previa inscripción en el Registro administrativo de buques y empresas navieras) (arts. 149 y 151) al que no tienen acceso estos buques fluviales porque los encargados de tal registro no tienen jurisdicción sobre tales aguas. 


\section{Derecho de navegación y noción de naviero}

El Derecho Marítimo, en nuestro Ordenamiento jurídico, en opinión común, no es sino el Derecho Privado de la navegación empresarial marítima. Pero hay otro modo de entender las cosas al que están la mayor parte de los autores que estudian el Derecho Marítimo: afirmar su autonomía como una rama del Derecho que comprende y requiere unas instituciones y principios propios y un tratamiento diferente de las instituciones comunes; e, incluso, como una disciplina en la que han de integrarse normas de Derecho Privado y de Derecho Público. Una autonomía que tuvo el Derecho Marítimo históricamente (aun en lo que hace a sus fuentes de manifestación), que perdió en parte en la época codificadora y que ahora ha recobrado en determinados países, como es el caso de Italia con el Código de la Navegación y de Francia por la promulgación de diversas leyes especiales.

Un tema, el anterior, que será obligado resolver a la hora de modificar el libro tercero del Código de Comercio.

En cualquier caso, y a diferencia de lo que pudo ser en otra época, ha de tenerse en cuenta la realidad actual, en la que son usuales, junto a la navegación con fines de transporte de mercancías y pasajeros y a la navegación de guerra, otras empresariales y no empresariales (de remolque, pesca, de recreo, de investigación, de pesca deportiva...) Más aún, con el progreso de la tecnología, el buque no es sino una de las muchas construcciones que pueden encontrarse en la mar, donde cada vez son más frecuentes otras tales como las plataformas petrolíferas, oleoductos, pontones, diques flotantes, hoteles flotantes... De ahí, que se haya dicho que el Derecho Marítimo debe ampliar su ámbito de aplicación y regular las actividades de navegación y, en general, todo este conjunto de relaciones que nacen o se desarrollan en el mar.

En consecuencia, si se opta por configurar el Derecho Marítimo como un Derecho de la navegación por mar (o por atribuirle ese contenido aún más amplio anteriormente indicado), para fijar la noción de naviero, habrá que tener en cuenta que la navegación actual no es en todo caso una navegación empresarial; y entender, como el Código de la Navegación (art. 265), que el naviero no es en todo caso un empresario marítimo, sino la persona que lleva a efecto, o aquélla en cuyo nombre se lleva a efecto, una actividad de navegación con uno o varios buques; sin perjuicio de que no le sea aplicable en todo caso el mismo régimen jurídico y de la noción de buque que es propia de este concepto.

\section{Respondabilidad del naviero}

Del Régimen Jurídico aplicable al naviero no vamos a ocuparnos extensamente, realmente el tema debe ser objeto de un estudio separado. 
No haremos sino unas breves referencias a la responsabilidad del naviero y del propietario en sus aspectos básicos, sin estudiar la limitación de la responsabilidad, también por la razón que se acaba de indicar.

\section{En el Código de Comercio}

1. En la interpretación del Código de Comercio, es opinión doctrinal generalizada entender que el naviero asume las obligaciones y responsabilidades que puedan derivar de la explotación del buque en la esfera contractual y extracontractual: una consecuencia, se dice, lógica, porque el naviero es el empresario marítimo; y que el propietario sólo es responsable excepcionalmente, como se dijo; sistema que se entiende fundado en lo que hace a la norma general y no por lo que se refiere a la excepción, porque el propietario no debe responder (y se dice incluso que la excepción hay que entenderla aplicable únicamente cuando el propietario sea naviero).

Una interpretación que se hace con base principalmente en los siguientes preceptos: los arts. 586 y 588, sobre responsabilidad del naviero por las obligaciones contraídas por el capitán; los arts. 587, 618 y 619, por lo que se refiere la responsabilidad del naviero por los actos del capitán, el primero con relación a los daños al cargamento; los arts. 826 y ss. para los casos de abordaje... Y, por lo que hace al propietario (y se ha indicado), los arts. 586 y 588.

Un sistema, sin embargo, que no es completo, porque no contiene una norma general; ni se refiere sino a las responsabilidades por los actos del capitán y en ciertas situaciones excepcionales como las de abordaje, naufragio...; y, por eso, que se entiendan aplicables las normas generales del Código de Comercio al regular la actuación de los auxiliares dependientes y comisionistas (art. 253 y 281 y ss.) y las del Código Civil que hacen a los dueños o directores de un establecimiento o empresa responsables por los perjuicios causados por sus dependientes en los ramos en que los tuvieren empleados o con ocasión de sus funciones (arts. $1.902 \mathrm{y}$ 1.903); las primeras en el orden contractual, éstas en el extracontractual.

Pues bien, ante la reforma del libro tercero del Código de Comercio, las líneas generales del sistema deben ser las siguientes:

La norma general habrá de ser, como lo es actualmente, la responsabilidad general del naviero.

Por lo que hace al propietario, habrá que diferenciar y distinguir dos situaciones: en primer lugar, aquéllas excepcionales en que el propietario es deudor y responsable, como son las de remoción de restos, pago de premios de asistencia o salvamento... y contaminación por vertido de hidrocarburos, ésta a virtud de una presunción de autoría a que están las 
normas internacionales (Convenio de Bruselas de 29 de noviembre de 1969); y, de otro; las de afección real del buque, de responsabilidad sin deuda, de responsabilidad limitada al buque; esto es, las de afección real del buque ante los acreedores marítimos privilegiados, que habrán de ser ahora los que se recogen como tales en el Convenio internacional firmado en Ginebra el 19 de abril de 1993. (Una actualización ésta que obligará a reformar el sistema registral).

Y habrá que modificar también la normativa aplicable a la limitación de responsabilidad para acomodarla al Convenio de Londres de 19 de noviembre de 1976, modificando el sistema, los supuestos..., creando un procedimiento adecuado para constituir el fondo de limitación, y ampliando las personas legitimadas que pueden limitar la responsabilidad, que han de ser el naviero y, también, el propietario, los auxiliares de aquél, las empresas que presten asistencia o salvamento al buque y la Compañía aseguradora.

\section{En el Código Penal}

Pero las normas del Código de Comercio y Código Civil no son las únicas a tener en cuenta para determinar la responsabilidad civil del naviero: en primer lugar, porque su responsabilidad puede derivar de un delito o falta, supuesto al que son aplicables las normas del Código Penal sobre responsabilidad civil por las infracciones penales (arts. 19 a 22) y sobre extensión de la responsabilidad civil (y costas) (arts. 101 a 111), y, ahora, al ser aplicables asimismo las normas sobre responsabilidad civil incluidas en la Ley de Puertos del Estado y de la Marina Mercante de 24 de noviembre de 1992 (dictadas, parece, para las situaciones de infracción administrativa que tipifica).

Derogada la Ley Penal y Disciplinaria de la Marina Mercante, de 22 de diciembre de 1955, por la citada Ley de Puertos del Estado (disp. derog., 2 (i), los únicos preceptos penales aplicables son los generales del Código Penal,y, singularmente, en lo que hace a la navegación, los que tipifican los delitos y faltas cometidos por imprudencia (arts. 563, 565,586 bis y 600 ).

Pues bien, a tenor de las normas del Código Penal citadas en el primer párrafo, son responsables civiles no sólo las personas que lo sean penalmente (con las excepciones que se indican), sino también, aunque subsidiariamente, y, entre otros, «las personas o empresas por los delitos que se cometan en los establecimientos que dirijan» $\mathrm{y}$ «los organismos, entidades y empresas dedicadas a cualquier género de industria por los delitos o faltas en que hubiesen incurrido sus criados, discípulos, oficiales, aprendices, empleados o dependientes en el desempeño de sus obli- 
gaciones o servicio» (arts. 19 a 22). Preceptos de los que resulta la responsabilidad civil del naviero por los delitos que puedan ser cometidos por sus dependientes (y, entre éstos, por la tripulación); responsabilidad, que es aquí subsidiaria a diferencia del sistema del Código de Comercio y del Código Civil en que hay una responsabilidad directa.

En lo que hace a la cuantía de esta responsabilidad civil, el Código Penal contiene determinadas normas (arts. 103 y 104); y, sin embargo no hay que entender que la aplicación de estos preceptos, y el hecho de que la responsabilidad del naviero sea en general subsidiaria, ha de impedir la aplicación de la limitación de responsabilidad en los casos que proceda.

Pero el Código Penal va a ser reformado y hay un Ante-proyecto en fase de aprobación. Este Ante-proyecto está básicamente al sistema que hemos delineado (arts. 119 a 131) aunque con algunas modificaciones de las que hay que destacar las siguientes:

a) La responsabilidad civil subsidiaria del empresario, y, por tanto, la del naviero, viene ahora regulada en términos más estrictos en lo que hace a los delitos dolosos cometidos por los auxiliares y se exige la negligencia en la vigilancia de la actividad que desarrollen o de las aptitudes y condiciones personales (art. 121).

b) Sobre la responsabilidad civil subsidiaria se dispone expresamente que «tendrá la misma extensión que la del responsable principal en cuyo defecto sea exigible».

Una norma ésta que agrava el problema aludido sobre la posiblidad de que el naviero limite su responsabilidad en el proceso penal y que obliga a modificar el Código de Comercio para que admita expresamente la limitación de responsabilidad del capitán y tripulación del buque (y de la empresa aseguradora).

c) Por lo que hace a la responsabilidad civil del Estado y Entidades Públicas, se dice expresamente que no cabe la ejecución de la responsabilidad civil en el proceso penal (art.122) (una ejecución que no cabe tampoco actualmente).

\section{En la Ley de Puertos y de Marina Mercante}

Por su parte, la Ley de Puertos del Estado y de la Marina Mercante introduce, en lo que se refiere a la responsabilidad civil del naviero (y demás personas interesadas), un conjunto de normas fundamentales que cabe sintetizar en los siguientes términos:

1. Se obliga a los navieros españoles (y a los navieros de buques no abanderados en España que naveguen por aguas en que el Estado español 
ejerza algún tipo de soberanía o jurisdicción) a tener asegurada la responsabilidad civil en los términos que se declaren reglamentariamente (art. 78).

(Un precepto que, puesto en relación con los del Código Penal relativos a las imprudencias punibles, supondrá la despenalización de todas aquéllas en que el resultado sea de daños a las cosas cuando no superen los límites de tal seguro; un efecto que difícilmente puede entenderse aceptado expresa ni tácitamente por dicha Ley).

2. Las infracciones administrativas, derogada la Ley Penal y Disciplinaria de la Marina Mercante y despenalizadas en su mayor parte las imprudencias tras la reforma última del Código Penal, son ahora norma general. Unas infracciones que la Ley clasifica en cuatro categorías: relativas al uso del puerto e instalaciones; contra la seguridad marítima; contra la ordenación del tráfico marítimo, y relativas a la contaminación del medio marino, (arts. 114 a 119, éste de interés especial en lo que hace a la posible calificación de estas infracciones como delitos o faltas).

Estas infracciones, que lo son por acción u omisión, por negligencia, por infracción de normas legales o reglamentarias..., incluyen todo un conjunto extenso de supuestos de los que pueden resultar daños a las personas o a las cosas, éstos con independencia de la titularidad pública o privada de los bienes... y responsabilidad por los daños y perjuicios causados.

Y, así, y entre otras muchas, son infracciones administrativas expresamente tipificadas como tales:

El incumplimiento de las Ordenanzas establecidas o instrucciones dadas por las Autoridades portuarias en la ejecución de las operaciones marítimas; que el capitán no solicite los servicios de práctico o de remolcadores si éstos son obligatorios; cuando por negligencia o dolosamente se causan daños a las obras, instalaciones, mercaderías, contenedores o medios de transporte terrestres o marítimos situados en la zona portuaria.

Ordenar o emprender la navegación sin que el buque reúna las debidas condiciones de navegabilidad; que los navieros, capitanes o patrones incumplan las normas sobre reconocimiento de buques y certificados; si se infringen las disposiciones reglamentarias sobre seguridad y se ocasionan daños a las personas; cuando se navega fuera de los canales balizados de acceso a la costa.

Si se incumplen las instruciones de los Capitanes Marítimos en el ámbito de sus competencias en relación con las maniobras y navegación del buque en aguas portuarias y fuera de ellas..., y, de una forma generalizada, la contaminación del medio marino. 
3. Pues bien, en todos estos casos, la infracción supone como medida no sancionadora la indemnización de los daños «irreparables» por cuantía igual al valor de los bienes destruidos o del deterioro causado y de los perjuicios ocasionados (art. 121).

En la fijación de la indemnización, hay que estar a las siguientes reglas (que no coinciden con las del C.c.): cuando el beneficio que resulte para el infractor sea superior al daño, la cuantía de la indemnización será al menos por el importe de aquél (arts. 121 y 124); cuando los daños sean de difícil evaluación, se aplicará, entre los criterios siguientes, el que suponga un mayor valor: coste teórico de reposición y restitución, valor de los bienes dañados y beneficio obtenido por el infractor (art. 124).

Unas indemnizaciones, en fin, que, sin reserva de ningún tipo, pueden ser exigidas por la vía administrativa de apremio (art.126); una norma que ha de ser justamente interpretada y entenderla referida únicamente al supuesto de que los intereses dañados sean interés de un Ente Público.

4. En el caso de estas infracciones administrativas, las personas responsables de las sanciones lo son también de la obligación de indemnizar los daños y perjuicios causados (arts 118 y 124). Estas personas responsables están determinadas en una pluralidad de reglas, que en lo esencial son las siguientes:

De las infracciones en materia de usos y actividades portuarias relacionadas con el buque, el naviero y solidariamente el consignatario o, en su defecto, el capitán... (art. 118. 1.b; una norma ésta que forma parte de un amplio conjunto).

De las infracciones en materia de marina civil cometidas con ocasión de la navegación marítima de buques mercantes, la empresa naviera titular de la actividad o, en su defecto, el capitán del buque.

En lo que se refiere a la navegación no empresarial o a las actividades empresariales que se desarrollen desde una plataforma fija, en el primer caso, la persona física o jurídica propietaria de la embarcación o la que sea directamente responsable de la infracción; y, en el segundo, la persona física o jurídica titular de la actividad empresarial.

De las infracciones por contaminación producida desde un buque (excediendo lo que es norma general en los Convenios internacionales), solidariamente, el naviero, el propietario, el asegurador de la responsabilidad civil y el capitán.

Estas infracciones administrativas, en determinados casos, pueden suponer la imposición de determinadas medidas complementarias específicas no siempre sancionadoras, como las siguientes:

En los casos de infracciones muy graves (que lo son, entre otros supuestos, cuando se causan lesiones a alguna persona que cause baja por 
incapacidad laboral superior a siete días o daños o perjuicios materiales superiores al millón de pesetas), la retención del buque o que se le impida su entrada o las operaciones de carga y descarga; en materia de contaminación del medio marino y como medida cautelar a fin de asegurar las que la Administración puede adoptar, la inmediata retención del buque, que podrá ser sustituida por aval o garantía suficiente a juicio de la Dirección General de la Marina Mercante, y como medida no sancionadora, cuando legal o reglamentariamente así se establezca, la denegación de escala, salida, carga o descarga del buque.

Pero estas normas dictadas para los casos de infracción administrativa no son las únicas de interés, pues hay otras específicas para los casos de peligro de hundimiento o de hundimiento. En el primer caso, la responsabilidad es del naviero o consignatario; en el segundo, de los propietarios, navieros, representantes o compañías aseguradoras (arts ...).

Ante este conjunto de normas, no siempre concordantes ni procedentes, la conclusión es, que hay que modificar y completar la normativa del Código de Comercio, pero también, y en lo necesario, la de otras leyes, como el Código Penal y la Ley de Puertos del Estado y de la Marina Mercante, para establecer un régimen jurídico actualizado y coherente. 